\title{
A Linked List Approach for Handwritten Textline Segmentation
}

\author{
C. Naveena and V. N. Manjunath Aradhya
}

\begin{abstract}
Textline segmentation in handwritten documents is of real challenge and interesting in the field of document image processing. In this paper, we propose a handwritten textline segmentation scheme based on the concept of linked list. The proposed method consists of three stages, namely, preprocessing, linked list and mathematical morphology. The concept of the linked list approach is used to build a textline sequence and mathematical morphology is used to obtain the line separator. We experimentally evaluated our proposed method on a document containing handwritten Kannada script. The results are compared with recent methods and show encouraging results.
\end{abstract}

Keywords. Textline Segmentation, Connected Component Analysis, Linked List, Mathematical Morphology.

2010 Mathematics Subject Classification. 68U10.

\section{Introduction}

Textline segmentation is one of the important preprocessing tasks for document image analysis. It provides crucial information for word/character segmentation and text recognition. Textline detection techniques are successful mainly in printed documents. However, processing of handwritten documents has been a key problem in the domain of document image analysis. Textline segmentation of handwritten documents is a complex and diverse problem. Thus, posing a challenge while handwritten document image processing.

Existing line extraction techniques can be categorized as global projection based on local grouping and smearing or on Hough-based methods. These projection based methods have been successfully applied for machine-printed documents. Variation in baseline and local orientation make them less effective for handwritten lines. Hough-based methods can handle documents with variations in orientation between textlines, but their performance also degrades rapidly when the baseline is not straight. Grouping approaches use connected components (CC) to handle complex layouts, but due to the proximity or touching characters across and within textlines, these methods turn out to be inadequate [2]. 
Many works have been done on English, Chinese, and Arabic language handwritten texts. However, some preliminary work has also been done on Indian scripts. Louloudis et al. [5] presented a textline detection of handwritten documents. The method describes block based Hough transform. A textline segmentation by clustering with distance metric learning is proposed in [12]. The method is based on minimal spanning tree clustering with distance metric learning techniques. A steerable directional local profile technique for extraction of handwritten Arabic textlines is described in [11]. The approach is based on the generalized adaptive local connectivity map (ACLM) using a steerable directional filter. A steerable filter is used to determine foreground intensity along multiple directions at each pixel while generating the ALCM. Yin and Liu [13] proposed a variational Bayes method for handwritten textline segmentation. The method is based on the Gaussian component and variational Bayes technique.

A handwritten document image segmentation into texlines and words is presented in [10]. The approach is based on the Viterbi algorithm. The algorithm tested handwriting segmentation contents on the benchmarking datasets of ICDAR-07. Du et al. [9] proposed a textline segmentation in handwritten documents using the Mumford shah model. The method used morphing to remove overlaps between textlines and connected broken ones. Liwicki et al. [4] proposed combining diverse on-line and off-line systems for handwritten textline recognition. The approach is based on multiple classifier system (MCS), hidden Markov models (HMMs) and bidirectional long short-term memory networks (BLSTM). A segmentation of Bangla unconstrained handwritten text is described in [8]. In this work, a horizontal histogram of stripes and the relationship of the minimal values of the histogram are used to extract a textline. A textline extraction from multi-skewed handwritten documents is presented in [3]. The method assumes that hypothetical water flows, from both left and right sides of the images, face obstruction from characters of textlines. The stripes areas left unwetted on the image frame are labeled for extraction of textlines.

Nagabhushan et al. [7] have described tracing and straightening the baseline in handwritten Persian/Arabic textline. The proposed technique identifies the candidate points from black and white blocks all along the textline. The algorithm traces the baseline using candidate points, which is subsequently stretched and straightened horizontally. Recently an elegant method for an unconstrained handwritten textline segmentation has been proposed by Alaei et al. [1]. The approach is based on the painting technique which enhances the separability between the foreground and background portions enabling for detection of textlines. Mohammadi et al. [6] described an improved handwritten textline segmentation technique. This method is an improvised method presented in [1]. In this method a dilation operation is 
used with respect to the slope of the line blocks instead of the simple horizontal dilation operation as used in [1].

From literature survey, it is clear that many works have been done on textline segmentation for English, Arabic, Chinese, Persian and also a few on Indian scripts. Even though many methods exist, compatible results for all languages have not been obtained so far. This is particularly true for South Indian languages like Kannada scripts. Kannada scripts textline extraction is a challenging task due to additional modifier-characters, which get plugged in as bottom fixes or top fixes, or as extensions that remain as disconnected protrusions of a main character. Under these circumstances, there is a need for developing a generalized approach which works for this kind of script.

This paper is organized as follows: In Section 2, the proposed method is described. Section 3 presents the experimental results and comparative study. The conclusion and future works are drawn at the end.

\section{Proposed Method}

The proposed method is divided into three stages, namely the preprocessing, implementing linked list and performing mathematical morphology. The following subsections explain the stages.

\subsection{Preprocessing}

In this stage, all the components in a document image (Figure 1) are detected by connected component analysis (CCA). For a component $c_{i}$, its height and width are represented by $h_{i}$ and $w_{i}$, respectively. The formation of any meaningful sentence in any language requires grammatical symbols like dots, strokes, descenders, ascenders etc. These symbols are called punctuations (small components). But in textline segmentation, this type of characters leads to redundant segmentation lines that may produce improper textline segmentation. To overcome this type of problem, the small components whose box height is very small compared to a preset threshold value were removed, as shown in Figure 2. The selected components are then blocked, for the purpose of creating nodes (explained in Section 2.2) which is shown in Figure 3.

In Figure 3, some blocks appear to connect two consecutive textlines or having longer heights with respect to the heights of the other blocks. These blocks indicate overlapping portions. Generally, when two textlines touch/overlap, two or more consecutive blocks obtained from the joined two lines make a longer blocks. The height of this blocks will be greater or equal to twice the size of the average height 


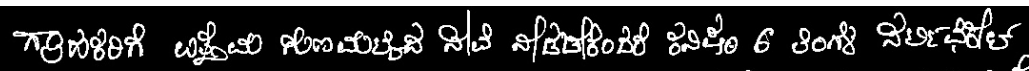

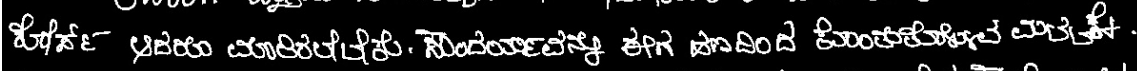
30.3

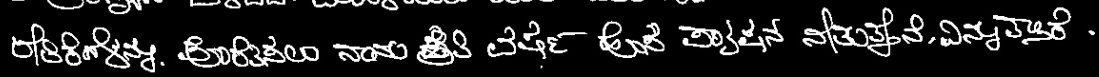

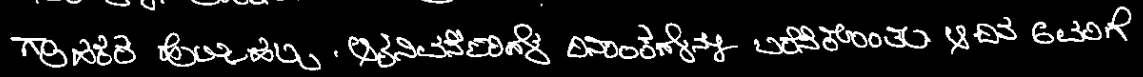

Figure 1. Sample image of handwritten Kannada document.

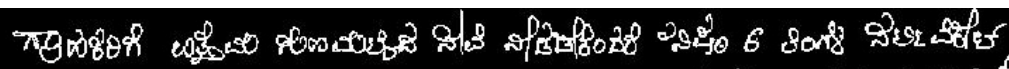

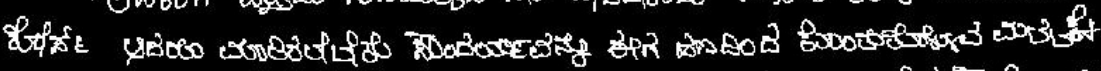

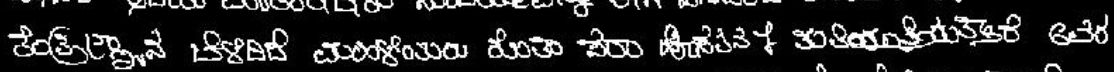

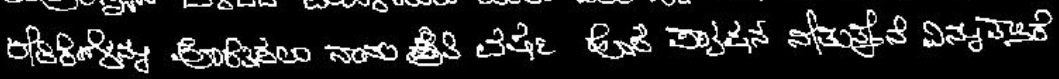

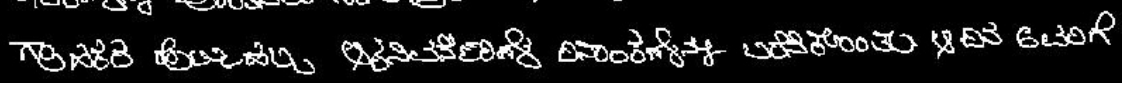

Figure 2. Result obtained after removing small components.

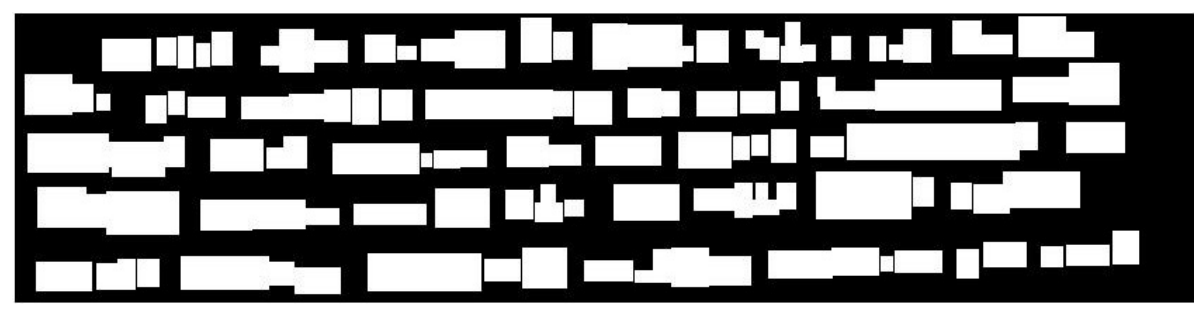

Figure 3. Document obtained after selected components are blocked.

of the blocks. All such longer blocks are removed temporarily, which is shown in Figure 4, and these blocks are used in the final stage of segmentation.

\subsection{Linked List}

Based on the concept of a node in a linked list, each block in Figure 4 is a node. Each textline nodes together represent a sequence. To build this sequences of textline, we find the center point $\left(R_{\text {mid }}\right)$ of the right part of the nodes using the equation (2) (see Figure 5). From the $R_{\text {mid }}$ point we draw a horizontal line with respect to the zero degree up to the line reach to the nearest neighbor node or up 


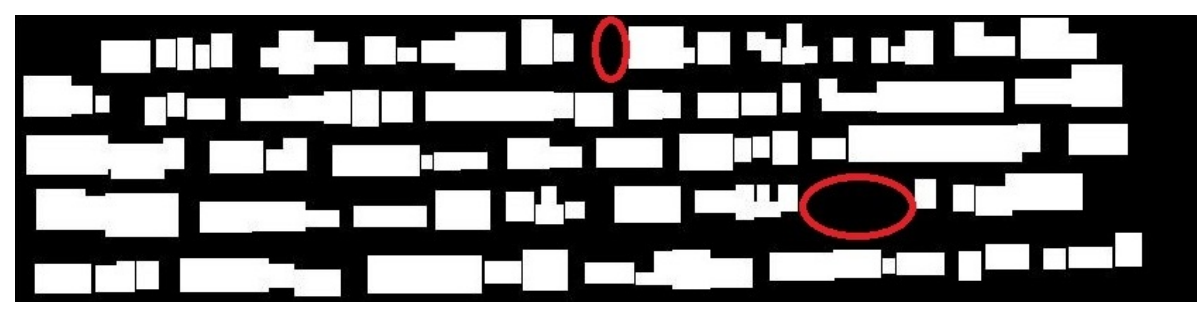

Figure 4. Result obtained after removing longer height blocks (shown in red color circle).

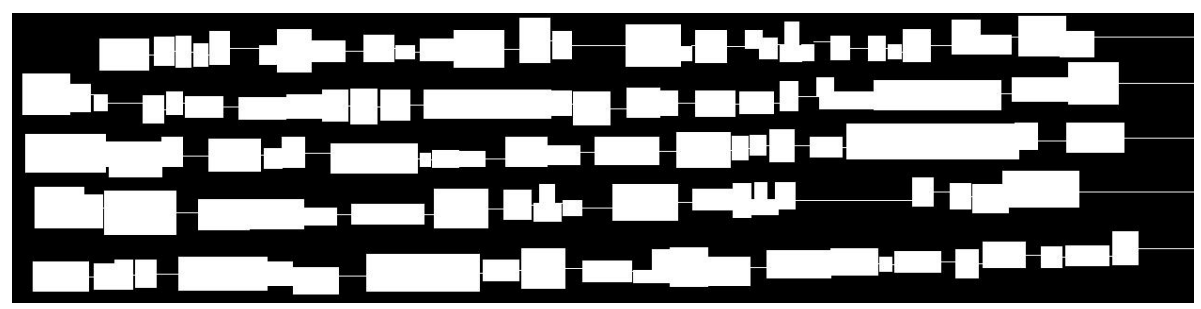

Figure 5. Result obtained after applying a linked list.

to the end of the text page which is shown in Figure 5.

$$
\begin{aligned}
\operatorname{mid} & =\frac{y_{\max }+y_{\min }}{2}, \\
R_{\text {mid }} & =\left(x_{\max }, \text { mid }\right) .
\end{aligned}
$$

\subsection{Mathematical Morphology}

In Figure 5, every sequence indicates the path information for final textline segmentation. Then the sequenced image is thinned by applying the morphological thinning algorithm to obtain textline separators which is shown in Figure 6. These line separators do not contain all the components in the textline because in the preprocessing stage (Section 2.1) we extracted longer height blocks. Hence, to obtain all the components, we added longer height blocks into the thinned image, which is shown in Figure 7, then after again we applied the thinning operation to obtain complete components of distinguished textline separators, which is shown in Figure 8. Finally, we add the components of the input image to the resultant complete components textline separator image (Figure 8). The result of final textline segmentation is shown in Figure 9. 


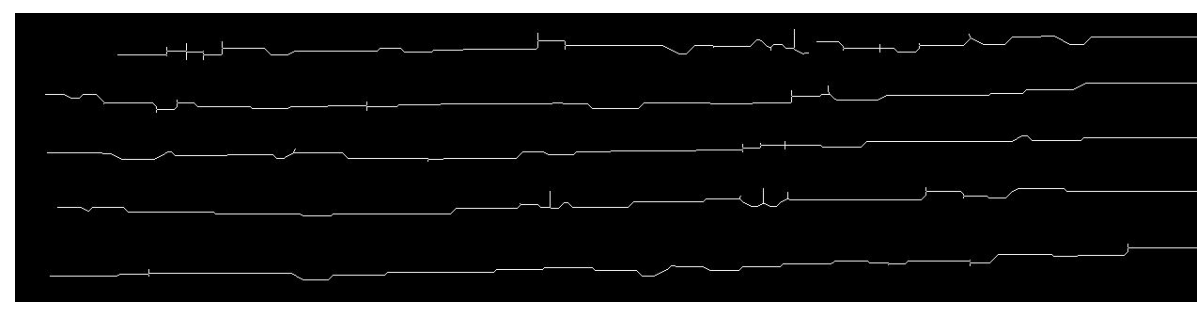

Figure 6. Result obtained after applying a thinning operation.

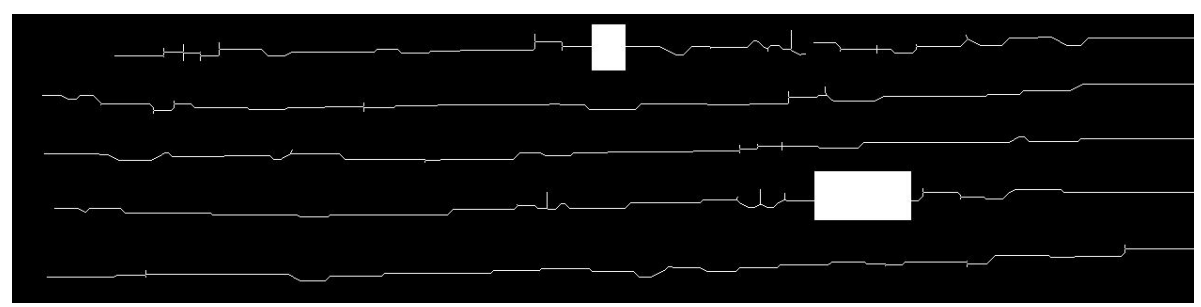

Figure 7. Result obtained after adding longer height blocks into the thinned image.

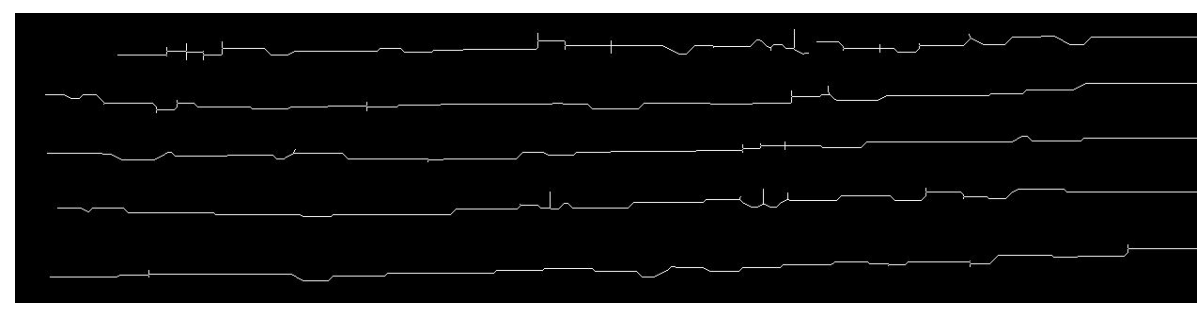

Figure 8. Result obtained after applying a thinning operation for Figure 7.

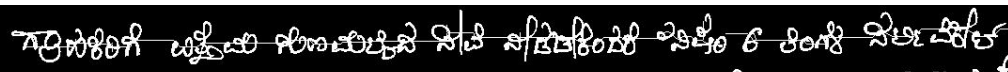

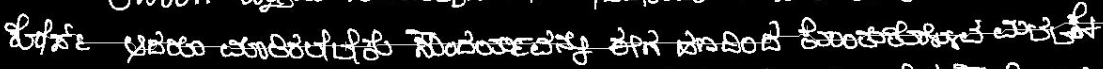

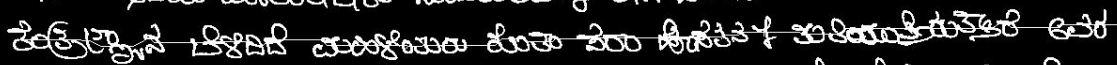

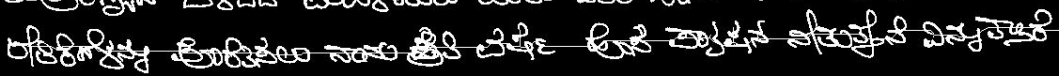

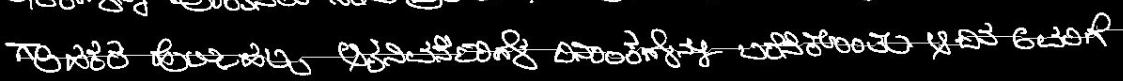

Figure 9. Result of the proposed textline segmentation algorithm. 


\section{Experiment Results and Comparative Study}

This section presents results of the experiment conducted to study the performance of the proposed method, which was implemented in MATLAB 10.0 on Core2Duo processor with 1 GB RAM. Over 100 Kannada handwritten document pages were considered from individuals including school children, undergraduate students, office employees etc. None of the documents includes any non-text elements and almost all documents have two or more adjacent textlines touching in several areas. Few documents have variable skew angles among textlines and having textlines with different skew directions as well as document images having textlines with converse skew angles along the same textline. For the experiment we considered single column document pages.

To evaluate the performance of the proposed method, we considered the recognition accuracy $(\mathrm{Rec})$ against the collected dataset.

The Rec measures as follows:

$$
\operatorname{Rec}=(\text { One2One } / N) \times 100,
$$

where One2One are complete segmented textlines in the documents, and $N$ is the total number of textlines presented in the documents.

The proposed method is compared with the recently proposed methods in [1] and [6]. Initially, we experimented the proposed method and achieved the recognition accuracy of $83.17 \%$. The error rate of the proposed method was a little high due to the textlines having touching components, which is shown in Figure 10. This problem has occurred due to the longer height blocks. In order to overcome this problem, we reduced the longer height blocks to $15 \%$ using mathematical erode operation. These reduced blocks are added to the thinned image (Figure 6) and the remaining procedure follows as explained in Section 2.3. After reducing longer height blocks, the recognition accuracy increased by 6\%. Figure 13 shows another example of successful results obtained for the proposed method. A comparison between the proposed method and existing methods is shown in Table 1. From Table 1, it is clear that the proposed method is better compared to the existing methods. The proposed method fails to extract textline for two cases, if the textlines having fluctuating nature and the textlines having more than 20 degree skew (which is shown in Figures 11 and 12). 


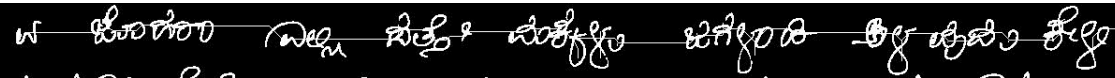

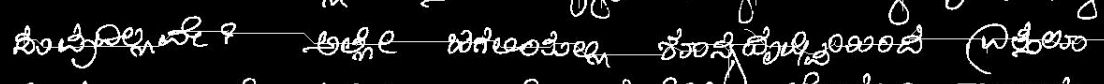

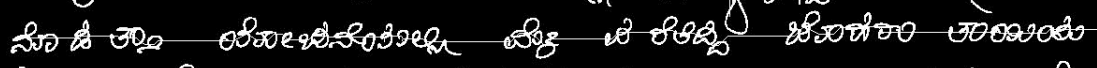

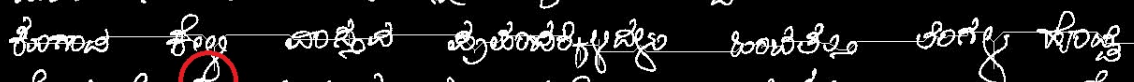

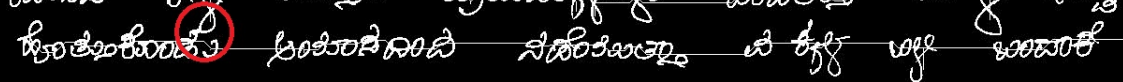

Figure 10. The textlines having touching components problem occurred during the conduct of our experiment.

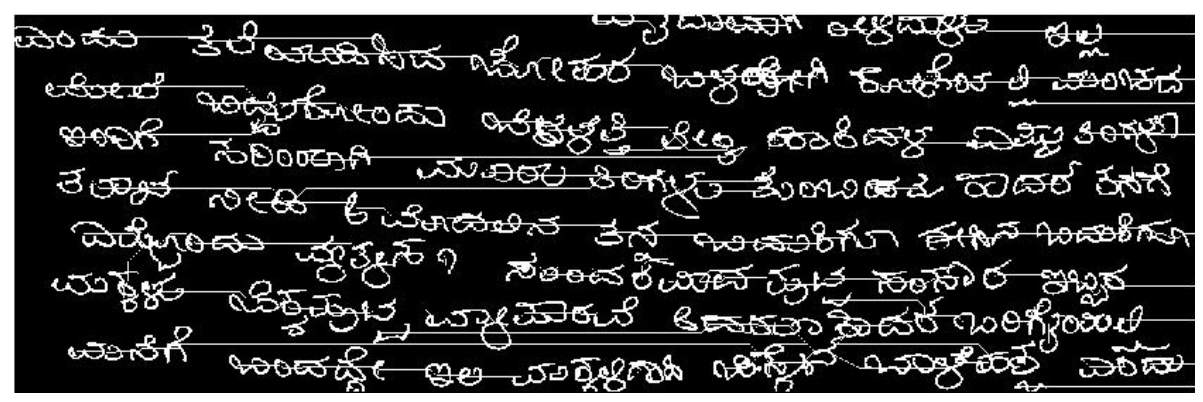

Figure 11. The proposed method fails to extract the textlines having fluctuating nature.

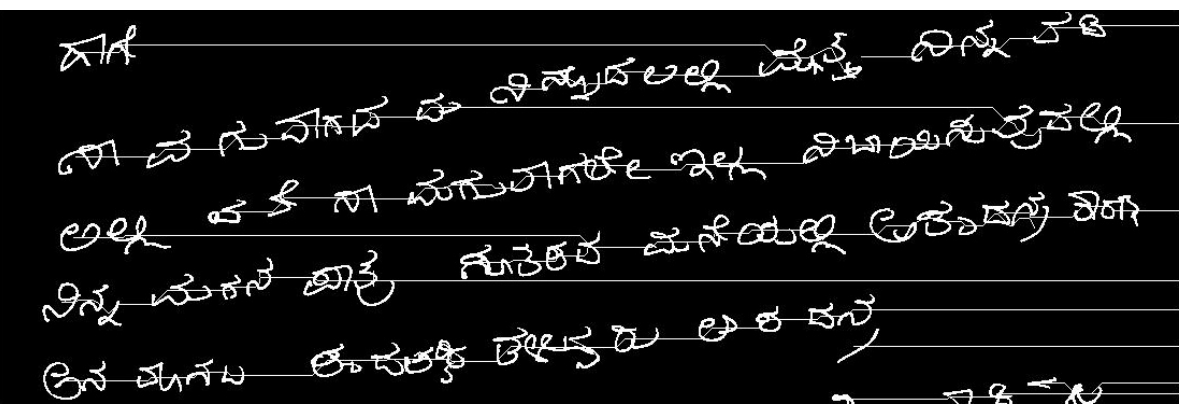

Figure 12. The proposed method fails to extract the textlines having more than 20 degree skew. 


\begin{tabular}{lccc}
\hline Methods & $N$ & One2One & Rec (in \%) \\
\hline PPA [1] & 617 & 449 & 72.89 \\
Improved PPA [6] & 617 & 502 & 81.3 \\
Proposed Method & $\mathbf{6 1 7}$ & $\mathbf{5 5 2}$ & $\mathbf{8 9 . 4}$ \\
\hline
\end{tabular}

Table 1. Recognition accuracy obtained for existing methods and proposed method.

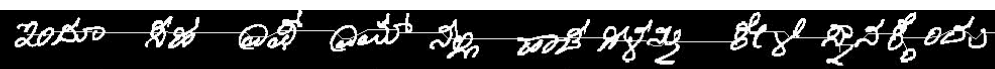

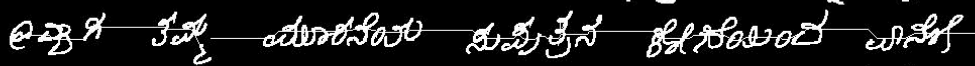

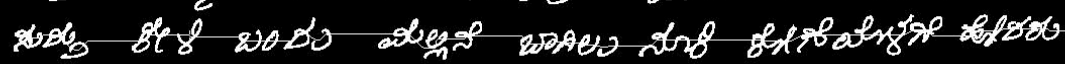

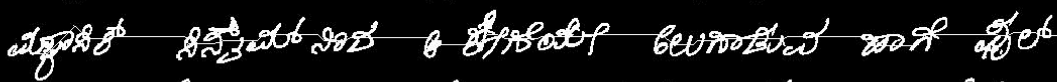

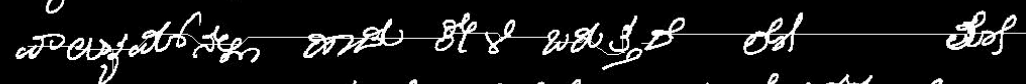

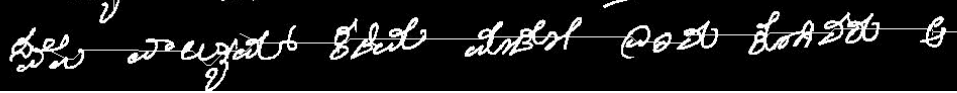

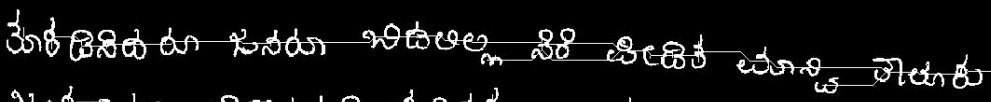

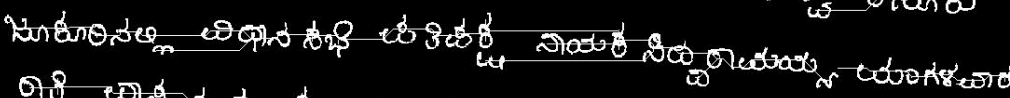

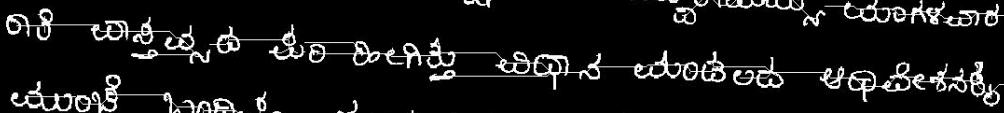

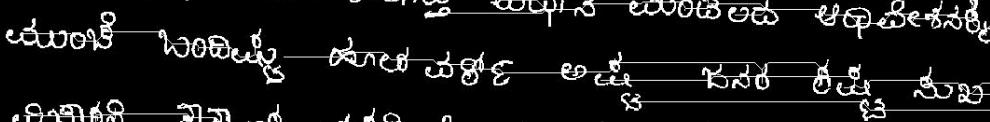

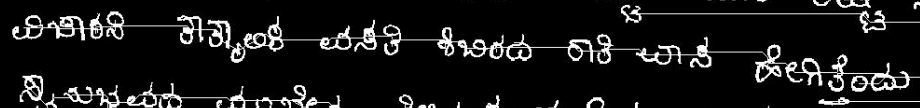

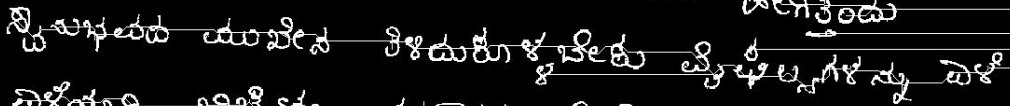

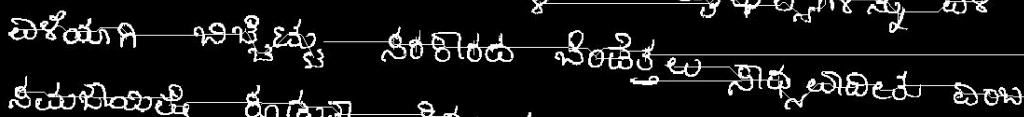

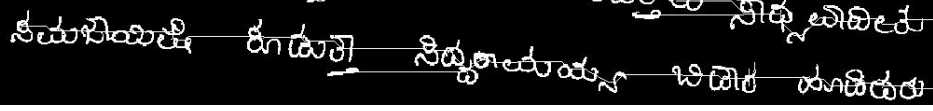

Figure 13. Successful results obtained for the proposed method. 


\section{Conclusion and Future Work}

In this paper, we proposed a textline extraction scheme for handwritten Kannada scripts. The proposed method involved three stages, the preprocessing, augmenting linked list and mathematical morphology. The proposed method was evaluated with unconstrained handwritten Kannada documents. The results were compared with well-known methods and showed encouraging results under different real time documents. The proposed method fails to detect fluctuating lines and textlines having a skew more than 20 degree. Efforts will be made to develop robust textline segmentation algorithm for handling texlines having more skew, fluctuating lines and over-segmentation problems.

\section{Bibliography}

[1] A. Alaei, U. Pal and P. Nagabhushan, A new scheme for unconstrained handwritten text-line segmentation, Patten Recognition 44 (2011), 917-928.

[2] J. Kumar, W. Abd-Almageed, L. Kang and D. Doermann, Handwritten Arabic text line segmentation using affinity propagation, in: Proceedings of the 8th IAPR International Workshop on Document Analysis Systems, ACM Press (2010), 135-142.

[3] M. Kundu, M. Nasipuri, S. Basu, C. Chaudhuri and D. K. Basu, Text line extraction from multi-skewde handwritten documents, Patten Recognition 40 (2007), 18251839.

[4] M. Liwicki and H. Bunke, Combining diverse on-line and off-line systems for handwritten text line recognition, Patten Recognition 42 (2009), 3254-3263.

[5] G. Louloudis, B. Gatos, I. Pratikakis and C. Halatsis, Text line detection in handwritten documents, Patten Recognition 41 (2009), 3758-3772.

[6] M. Mohammadi, S. S. M. Chanijani, V. N. M. Aradhya and G. H. Kumar, An improved handwritten text line segmentation technique, in: Proceedings of First International Conference on Advances in Computing and Communications (ACC 2011), Kochi, India, Springer (2011), 1534-1541.

[7] P. Nagabhushan and A. Alaei, Tracing and straightening the baseline in handwritten persian/arabic text-line: A new approach based on painting-technique, International Journal on Computer Science and Engineering 2 (2010), 907-916.

[8] U. Pal and S. Datta, Segmentation of Bangla unconstrained handwritten text, in: 7th International Conference on Document Analysis and Recognition, IEEE Computer Society (2003), 1128-1132.

[9] W. Pan, X. Du and T. D. Bui, Text line segmentation in handwritten documents using mumford shah model, Patten Recognition 42 (2009), 3136-3145. 
[10] V. Papavassiliou, T. S. Lakis, V. Kastsourous and G. Carayannis, Handwritten document image segmentation into text lines and words, Patten Recognition 43 (2010), 369-377.

[11] Z. Shi, S. Setlur and V. Govindaraju, A steerable directional local profile technique for extraction of handwritten arabic text lines, in: 10th International Conference on Document Analysis and Recognition, IEEE Computer Society (2009), 176-180.

[12] F. Yin and C. L. Liu, Handwritten text line segmentation by clustering with distance metric learning, in: Proceedings of the 11th ICFHR (2008), 229-234.

[13] F. Yin and C. L. Liu, A variational Bayes method for handwritten text line segmentation, in: 10th International Conference on Document Analysis and Recognition, IEEE Computer Society (2009), 436-440.

Received November 10, 2011.

\section{Author information}

C. Naveena, Department of ISE, Dayananda Sagar College of Engineering, Bangalore-78, India.

E-mail: naveena.cse@gmail.com

V. N. Manjunath Aradhya, Department of ISE, Dayananda Sagar College of Engineering, Bangalore-78, India.

E-mail: aradhya.mysore@gmail.com 